\title{
Effect of Nitrogenous and Bio-fertilization on Yield and Technological Characteristics of Some Wheat Varieties
}

\author{
Radwan, F. I. ${ }^{1}$, M.A. Gomaa', M.A. Ziton ${ }^{2}$ and A.M.M.Ahmed ${ }^{1}$ \\ ${ }^{1}$ Department of Agronomy, ${ }^{2}$ Department. of food Science and Technology \\ Faculty of Agriculture (Saba-Basha), Alexandria University
}

\begin{abstract}
Two field experiments were carried out at the experimental station farm Faculty of Agriculture (Saba -Basha), Alexandria University, Egypt, during 2013/2014 and 2014/2015 seasons to study the response of some wheat varieties (Triticum aestivum, L.) (Masr-1, Sakha-94 and Gemmeiza-9) and study the effect of nitrogen levels (zero, 35, 70 unit nitrogen) and biofertilization (Control, $35 \mathrm{Kg} / \mathrm{fed}+$ Microbein, $75 \mathrm{Kg} / \mathrm{fed}+$ Microbein) on yield and technological characteristics of wheat using spilt plot design with three replicates. The preceding summer crop was cotton in both growing seasons. The results could be summarized as follows Wheat cultivars, i.e. (Sakha-94, Masr-1 and Gemmeiza-9) were significantly differed in all growth characters under study. Nitrogen levels and Bio-fertilizers produced significant increments in all growth characters and Chemical composition. The interaction between wheat cultivars, nitrogen levels and biofertilizers showed significant effects on all studied traits. The obtained results showed the highest values plant height, number of tillers $\mathrm{m}^{-2}$, spike length, number of spike $\mathrm{m}^{2}$, number of spikelets/spikes, number of kernels/ spikes 1000-grain weight, spike weight, Ardabs, grain, straw and biological yields, harvest index, wheat moisture $\%$, ash\%, falling number sec, specific weight sec, total protein $\%$, wet gluten $\%$, dry gluten $\%$, gluten index $\%$, flour moisture $\%$, ash moisture\%, flour ash\%, bran ash\%, flour falling number sec, flour wet gluten\% and milling characters\%. Were obtained from the highest level of nitrogen $75 \mathrm{KG} / \mathrm{fed} \mathrm{N}$ and $75 \mathrm{Kg} / \mathrm{fed} \mathrm{N}+$ microbein.
\end{abstract}

Key world: Wheat varieties, Nitrogenous, bio-fertilizer, technological characteristics, yield.

\section{INTRODUCTION}

Wheat (Triticum aestivum L.) is the most widely cultivated cereal crop and is the major source of human food. The total production of wheat in Egypt reached 8.5 million tons in 2015 produced from an area of 3.7 million fedden (FAO. 2015). Ashmawy et al. (2010) found that the results cleared that Gemmeiza-9 cultivar surpassed each of Sakha-94 and Giza 168 cultivars in number of spikes $/ \mathrm{m}^{2}$, number of spikelets/spike, weight of 1000 grains, biological and grain yields in both seasons of study. Seleiman et al. ( 2010) found that Gimmeza-9 had the highest number of spikes per $\mathrm{m}^{2}$, number of grains of spike and grain yield. Rahimi (2010) studied the effect of nitrogen rates (0,40,60 and $80 \mathrm{~kg} \mathrm{~N} / \mathrm{ha})$ as urea and showed that effect of nitrogen on grain yield was highly significant. The grain yield was increased to $4800 \mathrm{~kg} / \mathrm{ha}$ by the application of $80 \mathrm{~kg} \mathrm{~N} / \mathrm{ha}$. Kandil et al. (2011) found that Bio-fertilizer treatments significantly affected plant height, tillers number $/ \mathrm{m}^{2}$, spikes number $/ \mathrm{m}^{2}$, number of grains/spike, grain weight/spike 1000 grain weight, biological yield, grain yield and straw yield, during the three seasons. Toaima et al. (2000) found that grain yield of Sids-1 variety. exhibited the highest percentage for all characteristics under study, Sakha-69 variety gave the lowest values and reported that hectoliter, flour and bran were significantly. Increased 
increasing nitrogen fertilization levels up to $80 \mathrm{~kg} / \mathrm{fed}$ and reported that the interaction effect of $\mathrm{N}$-levels with variety indicated that Sids-1 with application of 80 $\mathrm{kg} \mathrm{N} / \mathrm{fed}$. Increased ash, fiber, hectoliter and bran. Toaim et al. (2000) and ElNaggar (2003) found that Sakha-93 had the highest flour percentage and the lowest fine and coarse bran percentage, as compared with the other tested cultivars. Mahrous and Abd-Elhady (2006) reported that Gemmeiza-9 was most productive Egyptian cultivar. Kandil et al. (2011) found that Nitrogen fertilizer levels had significant effect on grain nitrogen percentage and crude protein content of wheat grain. Highest nitrogen fertilizer dose of $214.2 \mathrm{~kg} \mathrm{~N} / \mathrm{ha}$ significantly affected both grain nitrogen percentage and protein content which seemed to be interdependent since this highest nitrogen level induced the highest level of nitrogen percentage and crude protein of wheat grain. The highest means of grain protein percentage and crude protein content as an average of the three seasons was exerted when the higher nitrogen level of $214.2 \mathrm{~kg} \mathrm{~N} / \mathrm{ha}$ was applied. The lowest mean values of crude protein \% and nitrogen content were produced when the low nitrogen level of $107.1 \mathrm{~kg} \mathrm{~N} / \mathrm{ha}$ was applied.

It seems that nitrogen addition increased crude protein content as a result of increasing nitrogen availability and nitrogen uptake by plant. Ashmawy et al. (2010) found that the interaction of wheat cultivars and $N$ fertilization significantly affected spike length, biological and grain yields only in the first season. Also, indicated that spike length, biological and grain yields were significantly affected by the interaction between cultivars and N levels only in 2006/2007 season. However, the response of Gemmeiza-9 cultivar to the increase in $\mathrm{N}$ level was relatively different compared to the other two cultivars. The results in show that number of spikes $/ \mathrm{m}^{2}$, number of spikelet's/spike, number of grains/spike and weight of 1000 grains were not significantly affected by the interaction of cultivars and $\mathrm{N}$ fertilizer levels in both seasons of the study as well as spike length, biological and grain yields/fed in the second season.

Therefore objective of the present study was to evaluate the effect of nitrogen levels $46.5 \%$ and bio-fertilization on yield, with different conditioning times on Egypt wheat three properties (Masr-1, Sakha-94, Gemmeiza-9) and technological characteristics of some bread wheat varieties. Study the best class with a good technological property, Study the impact of mineral fertilizers to increase yield and facilitate the various elements of the plant and improve the quality of bread, Study the impact of bio-fertilization to provide hectic organisms of the soil and reduce pollution and increase technological properties of wheat and Study of the interaction between varieties and biological fertilization on wheat productivity and quality. 


\section{MATERALS AND METHODS}

Two field experiments were conducted at the experimental Farm, Faculty of Agriculture (Saba-Basha), Alexandria University, Egypt, during 2013/2014 and $2014 / 2015$ seasons. The experiments were carried out to study the effect of nitrogen levels $46.5 \%$ and bio-fertilization (Microbein) on yield and technological characteristics of some bread wheat varieties (Triticum aestivum, L.). Microbein as a commercial product were produced by Agriculture Research Center which includes Azotobacter spp., Azospirilhim spp. and Bacillus spp. The grain inoculation was done before sowing directly.

Each experiment was don using spilt-plot design in three replicates, where wheat cultivates (Masr-1, Sakha-94, Gemmeiza-9) occupied the main plot, while the nitrogen levels $(0,35$ and $75 \mathrm{~kg} / \mathrm{fed}$ with or without microbein) were assigned in the sub plots.

Cultivars were obtained from wheat Breeding Section Agriculture Research Center, Ministry of Agriculture, Egypt, and while Microbein obtained from wheat Breeding Section, Agriculture Research Center, Ministry of Agriculture, Egypt.

\section{Experimental Design}

Experimental design was split-plot with three replication included 18 treatments which were the combination between wheat cultivars and 6 treatments with nitrogen levels and Bio-fertilizers. Wheat cultivars were allocated in the main plots; both nitrogen and bio-fertilization were allocated in the sup-plots. The size of each plot was $6 \mathrm{~m}^{2}$ (2.0m long and $3.0 \mathrm{~m}$ wide). Sowing dates were November $21^{\text {th }}$ $2013 / 2014$ and $2014 / 2015$ in both seasons, respectively while seeding rate was 70 $\mathrm{kg} / \mathrm{fed}$. Calcium super phosphate $\left(15.5 \% \mathrm{P}_{2} \mathrm{O}_{5}\right)$ was applied during soil preparation at the rate of $37 \mathrm{~kg} \mathrm{ha}^{-1} \mathrm{P}_{2} \mathrm{O}_{5}$. Wheat growing samples were taken at random from each split-plot at different stages of growth, i.e. before heading stage (75 days after sowing), heading stage (90 days after sowing). First irrigation was applied at 25 days after sowing and then plants were irrigated every 25 days till the dough stage.

The preceding crop was cotton for the tow growing seasons, soil samples of the experimental sites were taken at the depth of zero to $15 \mathrm{~cm}$. and 15 to $30 \mathrm{~cm}$. from soil surface before the sowing. Physical and chemical analyses were done according to Chapman and Pratt (1978) at the Soil Laboratory of the Soil and Agricultural Chemistry Department, Faculty of Agriculture (Saba-Basha), Alexandria University. Detailed results of the soil characteristics are presented in Table (1). The experimental treatments can be described as follows:

\section{Nitrogen fertilization}

Nitrogen fertilizer was added in three doses at a rate of Control, $35 \mathrm{~kg} / \mathrm{fed}$, $75 \mathrm{~kg} / \mathrm{fed}$, Microbein, $35 \mathrm{~kg} / \mathrm{fed}+$ Microbein , $75 \mathrm{~kg} / \mathrm{fed}+$ Microbein N/fed. The 
recommended dose, Where, Control, $37.5 \mathrm{~kg} / \mathrm{fed}, 75 \mathrm{~kg} / \mathrm{fed}$, microbein, $37.5 \mathrm{~kg} / \mathrm{fed}$ + microbein, $75 \mathrm{~kg} / \mathrm{fed}+$ microbein were added at sowing time and first irrigation, $37.5,75 \mathrm{~kg} \mathrm{~N} / \mathrm{fed}$ added at the second irrigation (25 days after sowing). In the two experiments $\mathrm{N}$-fertilizers added in the form of ammonium nitrate $(46.5 \% \mathrm{~N})$.

\section{Bio-fertilization}

Microbein the bio fertilizer used in the present study produced by the Organization for Agriculture Equalization fund, Ministry of Agriculture, Egypt. It is prepared by adding equal amounts of these Microorganisms to carrier material and consists of a mixture of $\mathrm{N}_{2}$-fixing bacteria e.g. Azotobacter, chroococcum, Azospirillum brasilense and Bacillus polymyxa for Cerealin, while Biogien contain Azotobacter chroococcum only. The bio-fertilizer Microbein was obtained from wheat Breeding Section, Agriculture Research Center, Ministry of Agriculture.

Table (1). Soil chemical and mechanical analysis of the two experimental sites of $2013 / 2014$ and $2014 / 2015$ seasons.

\begin{tabular}{lcc}
\hline \multicolumn{1}{c}{ soil properties } & Season 1 (2013/2014) & Season 2 (2014/2015) \\
\hline $\mathrm{P}^{\mathrm{H}}$ & 8 & 7.9 \\
$\mathrm{EC}(\mathrm{dS} / \mathrm{m})$ & 1.73 & 1.74 \\
Total N(\%) & 0.35 & 0.4 \\
Organic matter (\%) & 1.8 & 1.75 \\
Available phosphate( $\mathrm{mg} / \mathrm{kg})$ & 3.4 & 3.2 \\
$\mathrm{Mg}++$ & 0.56 & 0.55 \\
$\mathrm{Na}+$ & 2.75 & 2.6 \\
$\mathrm{~K}+$ & 0.08 & 0.07 \\
$\mathrm{Ca}++$ & 0.92 & 0.9 \\
$\mathrm{CO}_{3}{ }^{+}+\mathrm{HCO}^{-}$ & 0.86 & 0.85 \\
$\mathrm{Cl}^{-}$ & 3.1 & 3 \\
$\mathrm{SO}_{4}{ }^{-}$ & 1.55 & 1.4 \\
$\mathrm{Sand}^{-}$ & 14.9 & 14.7 \\
$\mathrm{Silt}$ & 42.6 & 42.6 \\
$\mathrm{Clay}^{-}$ & 42.5 & 42.7 \\
$\mathrm{Soil} \mathrm{Texture}$ & Clay loam & Clay loam \\
\hline
\end{tabular}

\section{Collected Data}

Yield and yield components:

At the harvest time, one square meter was taken from each plot to determine the following: 
Spike Length (cm), Number of spikelet's / spike, Number of kernel / spike, Number of spikes $\mathrm{m}^{2}, 1000$ Kernel weight gm, Grains yield Ardab / fed, Straw yield ton / fed, Grain yield ton/fed, Harvest index (HI \%).

\section{The studied characteristics Chemical composition:}

Approximate composition was determined according to AOAC (2000). all analysis was carried out in triplicate.

Moisture according by AOAC (2000). Ash content according by AOAC (2000). Total protein cored by AOAC (2000). Gluten content cored by AOAC (2000). Milling experimental: Wheat samples (48 $\mathrm{kg}$, based on $14 \%$ moisture) were-tempered to $16 \%$ moisture content for 12,24 and $36 \mathrm{~h}$. prior to milling through a Buhler, MLU202, pneumatic mill by AACC method 26-31 (2000). Two transactions for wheat grinding were conducted of wheat flour are presented in Table (2). Roll spacing was set mechanically using a feeler gauge. Extraction rate of each treatment was calculated as follows: Extraction rate $=$ flour $(\mathrm{g}) /$ crude wheat $(\mathrm{g})^{*} 100$. Proximate chemical compositions

Table (2). Gap ( $\mathrm{mm})$ between roller mills.

\begin{tabular}{cccccc}
\hline Treatment & \multicolumn{2}{c}{ Break stream } & \multicolumn{2}{c}{ Reduction stream } \\
\hline & $\mathrm{B} 1$ & $\mathrm{~B} 2$ & $\mathrm{~B} 3$ & $\mathrm{C} 1$ & $\mathrm{C} 2$ \\
\hline Normal milling & 0.4 & 0.1 & 0.08 & 0.07 & 0.03 \\
\hline
\end{tabular}

\section{Statistical analysis}

Data were subjected to the proper statistical analysis as the technique of analysis of variance (ANOVA) of split plot design as mentioned by Gomez and Gomez (1984). Treatments were compared using the least Significant Difference (LSD) test as outline by Waller and Duncan (1969).

\section{Result and Discussion}

The data in Table (3) Indicted that growth attributes of wheat were significantly affected with different wheat varieties and nitrogen levels with biofertilization on both seasons. Wheat plant fertilized with $35 \mathrm{~kg} / \mathrm{fed}$ and $75 \mathrm{~kg} / \mathrm{fed} \mathrm{+}$ Microbein gave the highest values of plant height followed by those fertilized with $35 \mathrm{~kg} / \mathrm{fed}$ and $75 \mathrm{~kg} / \mathrm{fed}+$ Microbein,: simultaneously. Control fertilized and Microbein fertilized of height were the lowest. This was completely true in each of all studied characters. Sakha-94 and Gemmeiza-9 gave heights plant height on both seasons. The increases in plant height due to the increase in nitrogen with bio-fertilization. Number of tillers were significantly affected with different wheat varieties and nitrogen levels with bio-fertilization on both seasons. Sakha-94 gave heights number of tillers and Masr-1 gave the lowest on both seasons. Fertilized 
with $35 \mathrm{~kg} / \mathrm{fed}$ and $35 \mathrm{~kg} / \mathrm{fed}+$ Microbein $/ \mathrm{fed}$ gave the highest values Control fertilized and Microbein gave the lowest on both seasons. The data in Table (4) indicted that yield and its components. Sakha-94 gave heights number of spike $\mathrm{m}^{2}$, spike length and number spikelet's / spike. Masr-1 gave the lowest on number spikelets / spike, Gemmiza-9 and Masr-1 gave the lowest on number of spike $\mathrm{m}^{2}$, spike length. Fertilized with $75 \mathrm{~kg} / \mathrm{fed}$ and $75 \mathrm{~kg} / \mathrm{fed}+$ Microbein /fed gave the highest values on number of spike $\mathrm{m}^{2}$, spike length and number spikelet's / spike. And $35 \mathrm{~kg} / \mathrm{fed}+$ Microbein /fed gave the highest values zero unit /fed fertilized and Microbein unit /fed gave the lowest on both seasons. Ashmawy et al. (2010).found that The results cleared that Gemmeiza-9 cultivar surpassed each of Sakha-94 and Giza 168 cultivars in number of spikes $/ \mathrm{m}^{2}$, number of spikelet's/spike, weight of 1000 grains, biological and grain yields in both seasons of the study. Seleiman et al. (2010). found that Gimmeiza-9 had the highest number of spikes per $\mathrm{m}^{2}$, number of grains of spike and grain yield.

Significant differences were detected among the three tested cultivars for nitrogen levels and bio-fertilization on yield and yield components Table (5). Masr-1 gave heights on 1000 kernel weight, spike weight, Ardads ton/fed but Gemmeiza-9 and Sakha-94 gave the lowest on 1000 kernel weight and ardads ton/fed. Fertilized with 75 unit + Microben /fed gave the highest values on 1000 kernel weight, spike weight and ardads ton/fed on both seasons. Toaima et al. (2000). reported that the highest values of plant height, length and weight of spike, No. of spikes $/ \mathrm{m}^{2}$, No of grains / spike, weight of 1000-kernel and yield / fed. Were obtained from the highest levels of nitrogen $(80 \mathrm{~kg} / \mathrm{fed})$.

Table (6). Masr-1 gave heights on grain yield ton/fed, biological yield ton/fed, straw and HI\% but Gemmeiza- 9 and Sakha-94 gave the lowest. Fertilized with 75 unit + Microben /fed gave the highest values on grain yield ton/fed, biological yield ton/fed, straw and HI\% on both seasons. Control fertilized and Microben fertilized of height was the lowest. Abd El-Razek and El-Sheshtawy (2013). reported that the highest yield produced due to application of $\mathrm{N}$ rate of $180 \mathrm{~kg} \mathrm{ha}^{-1}$. And also, Kandil et al. (2011). Found that the highest yield was produced due to $\mathrm{N}$ level of $178.5 \mathrm{~kg} / \mathrm{ha}$. Both $\mathrm{N}$ and crude protein contents responded up to $214.5 \mathrm{~kg} \mathrm{~N} / \mathrm{ha}$. Also, Nitrogen levels had significant effect on plant height $(\mathrm{cm})$, number of tillers $/ \mathrm{m}^{2}$,spike number $/ \mathrm{m}^{2}$, number of grains/spike, grain weight/spike(g), 1000 grain weight ( $\mathrm{g}$ ), biological yield (t/ha), grain yield (ton/ha) and straw yield (t/ha). Also, Gafarr (2007). studied that effect of nitrogen fertilizer levels (0, 30, 60 and 90 $\mathrm{kg} \mathrm{N} / \mathrm{fed}$ ) on productivity of four wheat cultivars (Sids1, Sids7, Sakha-69 and Gemmeiza-9). He found the Gemmeiza9 gave the highest values of No. of kernel/spike, 1000-kernel weight, spike yield and protein percentage. 
Table (3). Effect of wheat varieties, nitrogenous and bio-fertilization on growth yield.

\begin{tabular}{|c|c|c|c|c|}
\hline & $\begin{array}{l}\text { plant height } \\
\text { Session1 }\end{array}$ & Session 2 & $\begin{array}{l}\text { N. of tillers m2 } \\
\text { Session } 1\end{array}$ & Session2 \\
\hline \multicolumn{5}{|l|}{ Varieties (V) } \\
\hline $\mathrm{V} 1$ & $86.61 \mathrm{~b}$ & $89.33 \mathrm{~b}$ & $304.33 \mathrm{~b}$ & $291.11 \mathrm{~b}$ \\
\hline $\mathrm{V} 2$ & $94.85 \mathrm{a}$ & $94.32 \mathrm{a}$ & $251 \mathrm{c}$ & $258.27 \mathrm{c}$ \\
\hline V3 & $95.17 \mathrm{a}$ & $95.12 \mathrm{a}$ & $375.33 \mathrm{a}$ & $390.66 \mathrm{a}$ \\
\hline LSD0.05 & 5.82 & 4.75 & 13.15 & 15.37 \\
\hline \multicolumn{5}{|l|}{ Fertilization (F) } \\
\hline $0 \mathrm{~N}$ & $74.54 \mathrm{~d}$ & $72.75 \mathrm{e}$ & $262.22 \mathrm{c}$ & $302.22 \mathrm{bcd}$ \\
\hline $35 \mathrm{~N}$ & $95.82 \mathrm{~b}$ & $96.75 \mathrm{c}$ & $314.44 a \mathrm{~b}$ & $274.66 \mathrm{~d}$ \\
\hline $75 \mathrm{~N}$ & $101.5 \mathrm{a}$ & $101.83 \mathrm{~b}$ & $314.66 a \mathrm{~b}$ & $334 \mathrm{ab}$ \\
\hline $0 \mathrm{~N}+$ Microbein & $80.74 \mathrm{c}$ & $80.46 \mathrm{~d}$ & $297.55 \mathrm{~b}$ & $330.66 \mathrm{abc}$ \\
\hline $35 \mathrm{~N}+\mathrm{M}$ & $98.18 \mathrm{a} b$ & $98.14 b \mathrm{c}$ & $343.77 \mathrm{a}$ & $339.66 \mathrm{a}$ \\
\hline $75 \mathrm{~N}+\mathrm{M}$ & $102.48 \mathrm{a}$ & $107.61 \mathrm{a}$ & $328.66 \mathrm{a} b$ & $298.66 \mathrm{~cd}$ \\
\hline LSD0.05 & 4.14 & 3.76 & 32.28 & 32.81 \\
\hline Interaction $\mathrm{V} * \mathrm{~F}$ & $* * *$ & $*$ & $* *$ & $* * *$ \\
\hline
\end{tabular}

Table (4). Effect of wheat varieties, nitrogenous and bio-fertilization on yield and its components.

\begin{tabular}{|c|c|c|c|c|c|c|c|c|}
\hline & \multicolumn{2}{|c|}{ N. spikelet's/spikes } & \multicolumn{2}{|c|}{ N. kernel spikes } & \multicolumn{2}{|c|}{ spike length cm } & \multicolumn{2}{|c|}{ N. of spike $\mathrm{m} 2$} \\
\hline & $\begin{array}{c}\text { Session } \\
1\end{array}$ & $\begin{array}{c}\text { Session } \\
2\end{array}$ & $\begin{array}{c}\text { Session } \\
1\end{array}$ & $\begin{array}{c}\text { Session } \\
2\end{array}$ & $\begin{array}{c}\text { Session } \\
1\end{array}$ & $\begin{array}{c}\text { Session } \\
2\end{array}$ & $\begin{array}{c}\text { Session } \\
1\end{array}$ & $\begin{array}{c}\text { Session } \\
2\end{array}$ \\
\hline \multicolumn{9}{|c|}{ Varieties (V) } \\
\hline V1 & $19.27 \mathrm{a}$ & $18.72 \mathrm{a}$ & $55.83 \mathrm{a}$ & $53.55 \mathrm{a}$ & $13.53 \mathrm{~b}$ & $14.63 \mathrm{~b}$ & $258.44 \mathrm{~b}$ & $244.5 \mathrm{~b}$ \\
\hline $\mathbf{V} 2$ & $18.33 \mathrm{~b}$ & $18.00 \mathrm{a}$ & $53.22 \mathrm{a}$ & $51.61 \mathrm{a}$ & $14.98 \mathrm{ab}$ & $13.98 \mathrm{~b}$ & $244.66 \mathrm{~b}$ & $195.77 \mathrm{c}$ \\
\hline V3 & $17.66 \mathrm{~b}$ & $17.27 \mathrm{a}$ & $52.0 \mathrm{a}$ & $50.33 \mathrm{a}$ & $16.09 \mathrm{a}$ & $15.96 \mathrm{a}$ & 339.77 a & $355.72 \mathrm{a}$ \\
\hline LSD0.05 & 0.89 & 1.63 & 3.87 & 5.29 & 1.53 & 1.09 & 34.85 & 40.37 \\
\hline \multicolumn{9}{|c|}{ Fertilization $(\mathbf{F})$} \\
\hline $\mathbf{0} \mathbf{N}$ & $16.88 \mathrm{c}$ & $14.77 \mathrm{e}$ & $48.44 \mathrm{c}$ & $41.44 \mathrm{e}$ & $12.26 \mathrm{~d}$ & $13.03 \mathrm{c}$ & $229.33 \mathrm{~d}$ & $232.88 \mathrm{~b}$ \\
\hline $35 N$ & $18.55 \mathrm{~b}$ & $18.66 \mathrm{ab}$ & $54.11 \mathrm{~b}$ & $53.66 \mathrm{bc}$ & $15.12 \mathrm{bc}$ & $14.73 \mathrm{~b}$ & $278.22 \mathrm{bc}$ & $220.44 \mathrm{~b}$ \\
\hline $75 N$ & $19.4 \mathrm{ab}$ & $19.77 \mathrm{ab}$ & $56.44 \mathrm{ab}$ & $57.33 \mathrm{ab}$ & $14.95 \mathrm{bc}$ & $17.38 \mathrm{a}$ & $290.66 \mathrm{~b}$ & $289.22 \mathrm{a}$ \\
\hline 0N+Microbein & $16.55 \mathrm{c}$ & $16.3 \mathrm{de}$ & $48.33 \mathrm{c}$ & $46.66 \mathrm{~d} \mathrm{e}$ & $13.75 \mathrm{~cd}$ & $14.17 \mathrm{bc}$ & $252.88 \mathrm{~cd}$ & $276.44 \mathrm{a}$ \\
\hline $35 \mathrm{~N}+\mathrm{M}$ & $18.88 \mathrm{ab}$ & $17.77 \mathrm{cb}$ & $55.22 \mathrm{ab}$ & $50.66 \mathrm{~cd}$ & $15.86 \mathrm{ab}$ & $13.61 \mathrm{bc}$ & $323.88 \mathrm{a}$ & $301.55 \mathrm{a}$ \\
\hline $75 N+M$ & $20.22 \mathrm{a}$ & $20.66 \mathrm{a}$ & $59.55 \mathrm{a}$ & $61.22 \mathrm{a}$ & $17.26 \mathrm{a}$ & $16.21 \mathrm{a}$ & $301.77 \mathrm{~b}$ & $271.44 \mathrm{a}$ \\
\hline LSD 0.05 & 1.39 & 1.799 & 4.16 & 5.62 & 1.6 & 1.34 & 26.96 & 29.53 \\
\hline $\begin{array}{c}\text { Interaction } \\
\mathbf{V}^{*} \mathbf{F}\end{array}$ & $\mathrm{ns}$ & $*$ & $\mathrm{~ns}$ & $*$ & $*$ & $\mathrm{~ns}$ & $* * *$ & $* * *$ \\
\hline
\end{tabular}


Table (5). Effect of nitrogenous, bio-fertilization and wheat varieties on yield and yield components

\begin{tabular}{|c|c|c|c|c|c|c|}
\hline & Weight & 1000 & spike & weight & Ardab & ton $\backslash$ fed \\
\hline & Session1 & Session2 & Session1 & Session2 & Session1 & Session2 \\
\hline \multicolumn{7}{|c|}{ Varieties $(\mathbf{V})$} \\
\hline V1 & $53.67 \mathrm{a}$ & $49.07 \mathrm{a}$ & $3.62 \mathrm{a}$ & $3.65 \mathrm{a}$ & $14.62 \mathrm{a}$ & $16.23 \mathrm{a}$ \\
\hline $\mathbf{V 2}$ & $47.71 \mathrm{~b}$ & $49.53 \mathrm{a}$ & $3.67 \mathrm{a}$ & $3.65 \mathrm{a}$ & $12.74 \mathrm{~b}$ & $14.38 \mathrm{~b}$ \\
\hline V3 & $49.52 \mathrm{~b}$ & $48.0 \mathrm{a}$ & $3.67 \mathrm{a}$ & $3.66 \mathrm{a}$ & $12.34 \mathrm{~b}$ & $13.42 \mathrm{c}$ \\
\hline LSD 0.05 & 2.08 & 1.72 & 0.46 & 0.2 & 0.61 & 0.4 \\
\hline \multicolumn{7}{|c|}{ Fertilization (F) } \\
\hline $\mathbf{0} \mathbf{N}$ & $41.43 \mathrm{e}$ & $40.47 \mathrm{~d}$ & $3.16 \mathrm{c}$ & $3.01 \mathrm{~b}$ & $8.98 \mathrm{f}$ & $9.67 \mathrm{e}$ \\
\hline $35 \mathrm{~N}$ & $47.96 \mathrm{c}$ & $46.33 \mathrm{c}$ & $3.58 \mathrm{~b}$ & $3.71 \mathrm{a}$ & $11.20 \mathrm{~d}$ & $13.31 \mathrm{c}$ \\
\hline $75 \mathrm{~N}$ & $56.57 \mathrm{a}$ & $52.47 \mathrm{~b}$ & $3.56 \mathrm{~b}$ & $3.67 \mathrm{a}$ & $16.06 \mathrm{~b}$ & $16.98 \mathrm{~b}$ \\
\hline $\mathbf{0} \mathbf{N}+$ Microbein & $44.34 \mathrm{~d}$ & $44.2 \mathrm{c}$ & $3.64 \mathrm{~b}$ & $3.61 \mathrm{a}$ & $10.62 \mathrm{e}$ & $11.78 \mathrm{~d}$ \\
\hline $35 \mathrm{~N}+\mathrm{M}$ & $53.94 \mathrm{~b}$ & $52.36 \mathrm{~b}$ & $3.94 \mathrm{ab}$ & $3.75 \mathrm{a}$ & $13.90 \mathrm{c}$ & $16.76 \mathrm{~b}$ \\
\hline $75 \mathrm{~N}+\mathrm{M}$ & $57.56 \mathrm{a}$ & $57.38 \mathrm{a}$ & $4.07 \mathrm{a}$ & $4.13 \mathrm{a}$ & $18.56 \mathrm{a}$ & $19.30 \mathrm{a}$ \\
\hline LSD 0.05 & 2.33 & 2.84 & 0.31 & 0.45 & 0.36 & 0.25 \\
\hline $\mathbf{V} * \mathbf{F}$ & $* * *$ & ns & $* *$ & ns & $* * *$ & $* * *$ \\
\hline
\end{tabular}

Significantly affected with different wheat varieties and nitrogen levels with bio-fertilization on chemical components Table (7) Masr-1 gave heights on Ash, falling number, specific weight. Sakha-94 gave heights protein. But Gemmiza-9 and Masr-1 gave the lowest on protein. Fertilized with $75 \mathrm{~kg}+$ Microbein gave the highest values on moisture, Ash, falling number, specific weight and protein on both seasons. Control fertilized and Microbein unit /fed fertilized of height were the lowest. Abd El-Rahim (1999). reported that falling number was $310 \mathrm{sec}$ for wheat flour (82\% extraction ratio) and $525 \mathrm{sec}$ for corn flour (97\% extraction ratio). AACC (2000). According that Ash, wet and dry gluten percentages, Crude protein percentage was obtained by multiplying grain nitrogen content.

Table (8) significantly affected with different wheat varieties and nitrogen levels with bio-fertilization on wheat gluten. Sakha-94 gave heights on wet, dry gluten; Masr-1 gave heights index gluten; Gemmiza-9 gave the lowest on both seasons. Fertilized with $75 \mathrm{~kg}+$ Microbein gave the highest values on wet, dry gluten and index gluten on both seasons. As reported by AACC (2000). According the method that Ash, wet and dry gluten percentages were measured and not agreed with Mahrous and Abd-Elhady (2006). reported that Gemmeiza-9 the most productive Egyptian cultivar. 
J. Adv. Agric. Res. (Fac. Agric. Saba Basha)

Table (6). Effect of wheat cultivars, nitrogen and bio-fertilization on yield and yield components

\begin{tabular}{|c|c|c|c|c|c|c|c|c|}
\hline & Grain & yield & Biological & yield & Straw & & HI \% & \\
\hline & $\begin{array}{c}\text { Session } \\
1 \\
\end{array}$ & $\begin{array}{c}\text { Session } \\
1 \\
\end{array}$ & $\begin{array}{c}\text { Session } \\
1 \\
\end{array}$ & $\begin{array}{c}\text { Session } \\
1 \\
\end{array}$ & $\begin{array}{c}\text { Session } \\
1 \\
\end{array}$ & $\begin{array}{c}\text { Session } \\
1 \\
\end{array}$ & $\begin{array}{c}\text { Session } \\
1 \\
\end{array}$ & $\begin{array}{c}\text { Session } \\
1 \\
\end{array}$ \\
\hline \multicolumn{9}{|c|}{ Varieties(V) } \\
\hline V1 & $2194.4 \mathrm{a}$ & $2434.8 \mathrm{a}$ & $4787.7 \mathrm{a}$ & $4743.8 \mathrm{~b}$ & $2593.57 \mathrm{a}$ & $2606.68 \mathrm{a}$ & $46.02 \mathrm{a}$ & $43.95 \mathrm{~b}$ \\
\hline V2 & $1912.3 \mathrm{~b}$ & $2156.7 \mathrm{~b}$ & $4155.1 \mathrm{~b}$ & $4833.6 \mathrm{ab}$ & $2243.81 \mathrm{~b}$ & $2636.51 \mathrm{a}$ & $45.45 \mathrm{a}$ & $45.8 \mathrm{a}$ \\
\hline V3 & $1851.2 \mathrm{~b}$ & $2014.3 \mathrm{c}$ & $3993.5 \mathrm{c}$ & $4962.0 \mathrm{a}$ & $2142.24 \mathrm{c}$ & $2690.69 \mathrm{a}$ & $45.30 \mathrm{a}$ & $45.7 \mathrm{a}$ \\
\hline LSD 0.05 & 106.3 & 77.85 & 113.22 & 185.1 & 33.81 & 114.95 & 1.16 & 0.77 \\
\hline \multicolumn{9}{|c|}{ Fertilization (F) } \\
\hline $\mathbf{0 N}$ & $1348.8 \mathrm{f}$ & $1453.33 \mathrm{e}$ & $3265.31 \mathrm{f}$ & $3468.82 \mathrm{f}$ & $1916.68 \mathrm{e}$ & $2015.48 \mathrm{f}$ & $41.23 \mathrm{~d}$ & $41.90 \mathrm{~d}$ \\
\hline $35 \mathrm{~N}$ & $1682.51 \mathrm{~d}$ & $2002.7 \mathrm{c}$ & $3748.84 \mathrm{~d}$ & $4484.83 \mathrm{~d}$ & $2068.45 \mathrm{~d}$ & $2482.13 \mathrm{~d}$ & $44.91 \mathrm{c}$ & $44.77 \mathrm{c}$ \\
\hline $75 N$ & 2410.46 b & $2541.66 \mathrm{~b}$ & $5100.87 \mathrm{~b}$ & $5623.55 \mathrm{~b}$ & $2690.50 \mathrm{~b}$ & $3081.88 \mathrm{~b}$ & $47.21 \mathrm{~b}$ & $45.42 \mathrm{c}$ \\
\hline 0N+Microbein & $1593.7 \mathrm{e}$ & $1762.02 \mathrm{~d}$ & $3580.67 \mathrm{e}$ & $3940.46 \mathrm{e}$ & $1987.04 \mathrm{e}$ & $2178.46 \mathrm{e}$ & $44.53 \mathrm{c}$ & $44.70 \mathrm{c}$ \\
\hline $35 \mathrm{~N}+\mathrm{M}$ & 2085.32 c & $2517.23 \mathrm{~b}$ & $4400.27 \mathrm{c}$ & $5397.18 \mathrm{c}$ & $2314.97 \mathrm{c}$ & $2879.98 \mathrm{c}$ & $47.37 \mathrm{ab}$ & $46.57 \mathrm{~b}$ \\
\hline $75 N+M$ & $2795.27 \mathrm{a}$ & $2935.03 \mathrm{a}$ & $5776.80 \mathrm{a}$ & $6164.71 \mathrm{a}$ & $2981.60 \mathrm{a}$ & $3229.81 \mathrm{a}$ & $48.3 \mathrm{a}$ & $47.60 \mathrm{a}$ \\
\hline LSD 0.05 & 52.38 & 44.68 & 102.91 & 107.48 & 78.34 & 96.48 & 0.92 & 0.97 \\
\hline $\mathbf{V} * \mathbf{F}$ & $* * *$ & $* * *$ & $* * *$ & $* * *$ & $* * *$ & $* * *$ & $* * *$ & $* * *$ \\
\hline
\end{tabular}

Table (7). Effect of wheat cultivars, nitrogen and bio-fertilization on chemical components on wheat

\begin{tabular}{|c|c|c|c|c|c|c|c|c|c|c|}
\hline & Wheat & $\begin{array}{c}\text { Moisture } \\
\%\end{array}$ & Ash & $\begin{array}{c}\text { Wwheat } \\
\%\end{array}$ & $\begin{array}{c}\text { Falling } \\
\text { number } \\
\text { wheat }\end{array}$ & (sec) & \multicolumn{2}{|c|}{ SP.WT } & \multicolumn{2}{|c|}{$\begin{array}{c}\text { Protein } \\
\%\end{array}$} \\
\hline & $\begin{array}{c}\text { Session } \\
1\end{array}$ & $\begin{array}{c}\text { Session } \\
2 \\
\end{array}$ & $\begin{array}{c}\text { Session } \\
1 \\
\end{array}$ & $\begin{array}{c}\text { Session } \\
2 \\
\end{array}$ & $\begin{array}{c}\text { Session } \\
1\end{array}$ & $\begin{array}{c}\text { Session } \\
2\end{array}$ & $\begin{array}{c}\text { Session } \\
1\end{array}$ & $\begin{array}{c}\text { Session } \\
2\end{array}$ & $\begin{array}{c}\text { Session } \\
1\end{array}$ & $\begin{array}{c}\text { Session } \\
2\end{array}$ \\
\hline \multicolumn{11}{|c|}{ Varieties(V) } \\
\hline V1 & $12.5 \mathrm{a}$ & $12.4 \mathrm{a}$ & $1.5 \mathrm{a}$ & $1.5 \mathrm{a}$ & $338.5 \mathrm{a}$ & $321.7 \mathrm{a}$ & $82.0 \mathrm{c}$ & $82.5 \mathrm{c}$ & $11.5 \mathrm{~b}$ & $10.7 \mathrm{c}$ \\
\hline V2 & $12.0 \mathrm{~b}$ & $12.2 \mathrm{~b}$ & $1.36 \mathrm{~b}$ & $179.8 \mathrm{c}$ & $213.5 \mathrm{c}$ & $213.5 \mathrm{c}$ & $86.5 \mathrm{a}$ & $86.3 \mathrm{a}$ & $10.8 \mathrm{c}$ & $11.0 \mathrm{~b}$ \\
\hline V3 & $12.5 \mathrm{a}$ & $12.0 \mathrm{~b}$ & $1.5 \mathrm{a}$ & $1.5 \mathrm{ab}$ & $272 \mathrm{~b}$ & $294.4 \mathrm{~b}$ & $84.4 \mathrm{~b}$ & $84.6 \mathrm{~b}$ & $12.5 \mathrm{a}$ & $12.0 \mathrm{a}$ \\
\hline LSD 0.05 & 0.13 & 0.21 & 0.05 & 0.13 & 16.9 & 9.4 & 0.69 & 1.2 & 0.14 & 0.27 \\
\hline \multicolumn{11}{|c|}{ Fertilization(F) } \\
\hline $\mathbf{0 N}$ & $12.3 \mathrm{abc}$ & $12.4 \mathrm{a}$ & $1.2 \mathrm{e}$ & $1.1 \mathrm{~d}$ & $192.4 \mathrm{e}$ & $190.8 \mathrm{e}$ & $80.8 \mathrm{~d}$ & $81.2 \mathrm{c}$ & $9.0 \mathrm{f}$ & $8.6 \mathrm{f}$ \\
\hline $35 \mathrm{~N}$ & $12.5 \mathrm{ab}$ & $12.3 \mathrm{ab}$ & $1.4 \mathrm{c}$ & $1.3 \mathrm{c}$ & $246 \mathrm{c}$ & $261 \mathrm{c}$ & $84.8 \mathrm{~b} \mathrm{c}$ & $83.7 \mathrm{~b}$ & $10.4 \mathrm{e}$ & $10.3 \mathrm{~d}$ \\
\hline $75 N$ & $12.3 \mathrm{bc}$ & $12.1 \mathrm{~b}$ & $1.6 \mathrm{a}$ & $1.6 \mathrm{~b}$ & $297.4 \mathrm{~b}$ & $306.4 \mathrm{~b}$ & $85.5 \mathrm{~b}$ & $86.0 \mathrm{a}$ & $12.1 \mathrm{c}$ & $12.8 \mathrm{~b}$ \\
\hline 0N+Microbein & $12.6 \mathrm{a}$ & $12.2 \mathrm{ab}$ & $1.3 \mathrm{~d}$ & $1.3 \mathrm{c}$ & $210.3 \mathrm{~d}$ & $229.6 \mathrm{~d}$ & $83.5 \mathrm{c}$ & $83.9 \mathrm{~b}$ & $10.8 \mathrm{~d}$ & $9.7 \mathrm{e}$ \\
\hline $35 \mathrm{~N}+\mathrm{M}$ & $12.4 \mathrm{abc}$ & $12.1 \mathrm{~b}$ & $1.5 \mathrm{~b}$ & $1.5 \mathrm{~b}$ & $304.3 \mathrm{~b}$ & $319.1 \mathrm{~b}$ & $84.8 \mathrm{bc}$ & $85.9 \mathrm{a}$ & $12.8 \mathrm{~b}$ & $12.1 \mathrm{c}$ \\
\hline $75 \mathrm{~N}+\mathrm{M}$ & $12.1 \mathrm{c}$ & $12.3 \mathrm{ab}$ & $1.6 \mathrm{a}$ & $1.8 \mathrm{a}$ & $331.6 \mathrm{a}$ & $351.5 \mathrm{a}$ & $87.4 \mathrm{a}$ & $86.2 \mathrm{a}$ & $14.2 \mathrm{a}$ & $13.8 \mathrm{a}$ \\
\hline LSD 0.05 & 0.25 & 0.21 & 0.07 & 0.11 & 12.28 & 15.54 & 1.6 & 0.9 & 0.23 & 0.33 \\
\hline $\begin{array}{c}\text { Interaction } \\
V^{*} \mathbf{F}\end{array}$ & $* * *$ & $* * *$ & $* * *$ & $* * *$ & $* * *$ & $* * *$ & $* * *$ & $* * *$ & $* * *$ & $* * *$ \\
\hline
\end{tabular}


Table (8). Different wheat varieties and nitrogen levels with bio-fertilization on wheat gluten

\begin{tabular}{|c|c|c|c|c|c|c|}
\hline & $\begin{array}{c}\text { Wet } \\
\text { Session1 }\end{array}$ & $\begin{array}{c}\text { gluten } \\
\text { Session2 }\end{array}$ & $\begin{array}{c}\text { Dry } \\
\text { Session1 }\end{array}$ & $\begin{array}{c}\text { gluten } \\
\text { Session2 }\end{array}$ & $\begin{array}{c}\text { Index } \\
\text { Session1 }\end{array}$ & Session2 \\
\hline \multicolumn{7}{|c|}{ Varieties(V) } \\
\hline V1 & $24.5 \mathrm{~b}$ & $22.5 \mathrm{~b}$ & $10.6 \mathrm{~b}$ & $10.0 \mathrm{~b}$ & $85 \mathrm{a}$ & $83.3 \mathrm{a}$ \\
\hline $\mathbf{V 2}$ & $20.9 c$ & $22.2 \mathrm{c}$ & $9.4 \mathrm{c}$ & $10.1 \mathrm{~b}$ & $82.0 \mathrm{~b}$ & $82.8 \mathrm{a}$ \\
\hline V3 & $25.3 \mathrm{a}$ & $24.6 \mathrm{a}$ & $14.3 \mathrm{a}$ & $14.4 \mathrm{a}$ & $80.6 \mathrm{c}$ & $80.5 \mathrm{~b}$ \\
\hline LSD 0.05 & 0.71 & 0.31 & 0.5 & 0.5 & 1.2 & 1.1 \\
\hline \multicolumn{7}{|c|}{ Fertilization $(\mathbf{F})$} \\
\hline $\mathbf{0} \mathbf{N}$ & $17.5 \mathrm{~d}$ & $17.3 \mathrm{f}$ & $8.2 \mathrm{e}$ & $7.9 \mathrm{e}$ & $76.5 \mathrm{~d}$ & $73.5 \mathrm{f}$ \\
\hline $35 \mathrm{~N}$ & $21.6 \mathrm{c}$ & $21.0 \mathrm{~d}$ & $10.4 \mathrm{c}$ & $10.0 \mathrm{~d}$ & $80.0 \mathrm{c}$ & $80.7 \mathrm{~d}$ \\
\hline $75 N$ & $26.1 \mathrm{~b}$ & $26.6 \mathrm{~b}$ & $12.8 \mathrm{~b}$ & $12.4 \mathrm{c}$ & $84.5 \mathrm{~b}$ & $84.3 \mathrm{c}$ \\
\hline 0N +Microbein & $21.7 \mathrm{c}$ & $19.9 \mathrm{e}$ & $9.7 \mathrm{~d}$ & $9.7 \mathrm{~d}$ & $79.3 \mathrm{c}$ & $78.3 \mathrm{e}$ \\
\hline $35 \mathrm{~N}+\mathrm{M}$ & $25.6 \mathrm{~b}$ & $24.9 \mathrm{c}$ & $12.9 \mathrm{~b}$ & $13.3 \mathrm{~b}$ & $85.6 \mathrm{~b}$ & $86.5 \mathrm{~b}$ \\
\hline $75 \mathrm{~N}+\mathrm{M}$ & $29.0 \mathrm{a}$ & $28.8 \mathrm{a}$ & $14.6 \mathrm{a}$ & $15.7 \mathrm{a}$ & $89.5 \mathrm{a}$ & $90.0 \mathrm{a}$ \\
\hline LSD 0.05 & 0.54 & 0.61 & 0.38 & 0.38 & 1.5 & 1.1 \\
\hline Interaction $\mathbf{V} * \mathbf{F}$ & $* * *$ & $* * *$ & $* * *$ & $* * *$ & $* * *$ & $* * *$ \\
\hline
\end{tabular}

Table (9). Effect of wheat cultivars, nitrogen and bio-fertilization on chemical components on wheat flour

\begin{tabular}{|c|c|c|c|c|c|c|c|c|c|c|c|c|}
\hline & $\begin{array}{c}\text { Flour } \\
\text { Session } \\
1 \\
\end{array}$ & $\begin{array}{c}\text { Moister } \\
\text { Session } \\
2 \\
\end{array}$ & $\begin{array}{c}\text { Bran } \\
\text { Session } \\
1 \\
\end{array}$ & $\begin{array}{c}\text { Moisture } \\
\text { Session } \\
2 \\
\end{array}$ & $\begin{array}{c}\text { Ash } \\
\text { Session } \\
1 \\
\end{array}$ & $\begin{array}{c}\text { flour } \\
\text { Session } \\
2 \\
\end{array}$ & $\begin{array}{c}\text { Ash } \\
\text { Session } \\
1 \\
\end{array}$ & $\begin{array}{c}\text { bran } \\
\text { Session } \\
2 \\
\end{array}$ & $\begin{array}{c}\text { Falling } \\
\text { Session } \\
1 \\
\end{array}$ & $\begin{array}{c}\text { number } \\
\text { Session } \\
2 \\
\end{array}$ & $\begin{array}{c}\text { Wet } \\
\text { Session } \\
1 \\
\end{array}$ & $\begin{array}{c}\text { gluten } \\
\text { Session } \\
2 \\
\end{array}$ \\
\hline \multicolumn{13}{|c|}{ Varieties $(V)$} \\
\hline V1 & $14.2 \mathrm{a}$ & $14.0 \mathrm{~b}$ & $13.8 \mathrm{a}$ & $13.6 \mathrm{~b}$ & $0.53 \mathrm{~b}$ & $0.6 \mathrm{a}$ & $0.30 \mathrm{~b}$ & $0.49 \mathrm{a}$ & $335.1 \mathrm{a}$ & $316.6 \mathrm{a}$ & $24.15 \mathrm{~b}$ & $22.3 \mathrm{~b}$ \\
\hline $\mathbf{V 2}$ & $14.22 \mathrm{a}$ & $14.34 \mathrm{a}$ & $13.82 \mathrm{a}$ & $13.94 \mathrm{a}$ & $0.53 \mathrm{~b}$ & $0.46 \mathrm{c}$ & $0.30 \mathrm{~b}$ & $0.34 \mathrm{c}$ & $184.8 \mathrm{c}$ & $223.7 \mathrm{c}$ & $20.75 \mathrm{c}$ & $21.92 \mathrm{c}$ \\
\hline V3 & $14.50 \mathrm{a}$ & $14.20 \mathrm{~b}$ & $14.10 \mathrm{a}$ & $13.81 \mathrm{a} \mathrm{b}$ & $0.60 \mathrm{a}$ & $0.51 \mathrm{~b}$ & $0.36 \mathrm{a}$ & $0.39 \mathrm{~b}$ & $279.5 b$ & $304.9 \mathrm{~b}$ & $25.02 \mathrm{a}$ & $24.11 \mathrm{a}$ \\
\hline LSD 0.05 & 0.31 & 0.22 & 0.31 & 0.22 & 0.017 & 0.015 & 0.018 & 0.016 & 14 & 8.5 & 0.19 & 0.16 \\
\hline \multicolumn{13}{|c|}{ Fertilization(F) } \\
\hline $\mathbf{0} \mathbf{N}$ & $14.17 \mathrm{a}$ & $14.0 \mathrm{a}$ & $13.7 \mathrm{a}$ & $13.6 \mathrm{a}$ & $0.42 \mathrm{~d}$ & $0.42 \mathrm{e}$ & $0.19 \mathrm{~d}$ & $0.29 \mathrm{e}$ & $195 \mathrm{e}$ & $196 \mathrm{f}$ & $17.4 \mathrm{e}$ & $17.35 \mathrm{f}$ \\
\hline $35 \mathrm{~N}$ & $14.35 \mathrm{a}$ & $14.12 \mathrm{a}$ & $13.95 \mathrm{a}$ & $13.70 \mathrm{a}$ & $0.47 \mathrm{c}$ & $0.48 \mathrm{c}$ & $0.24 \mathrm{c}$ & $0.35 \mathrm{c}$ & $247.2 \mathrm{c}$ & $267.6 \mathrm{~d}$ & $21.28 \mathrm{~d}$ & $20.6 \mathrm{~d}$ \\
\hline $75 \mathrm{~N}$ & $14.30 \mathrm{a}$ & $14.33 \mathrm{a}$ & $13.91 \mathrm{a}$ & $13.93 \mathrm{a}$ & $0.61 \mathrm{~b}$ & $0.58 \mathrm{~b}$ & $0.38 \mathrm{~b}$ & $0.45 \mathrm{~b}$ & $299.6 \mathrm{~b}$ & $301.5 \mathrm{c}$ & $25.68 \mathrm{~b}$ & $26.4 \mathrm{~b}$ \\
\hline 0N+Microbein & $14.51 \mathrm{a}$ & $14.12 \mathrm{a}$ & $14.10 \mathrm{a}$ & $13.7 \mathrm{a}$ & $0.44 \mathrm{~d}$ & $0.46 \mathrm{~d}$ & $0.21 \mathrm{~d}$ & $0.33 \mathrm{~d}$ & $21.5 \mathrm{~d}$ & $243.5 \mathrm{e}$ & $21.52 \mathrm{c}$ & $19.72 \mathrm{e}$ \\
\hline $35 \mathrm{~N}+\mathrm{M}$ & $14.26 \mathrm{a}$ & $14.27 \mathrm{a}$ & $13.86 \mathrm{a}$ & $13.87 \mathrm{a}$ & $0.63 \mathrm{~b}$ & $0.57 \mathrm{~b}$ & $0.39 \mathrm{~b}$ & $0.54 \mathrm{~b}$ & $307.8 \mathrm{~b}$ & $322.8 \mathrm{~b}$ & $25.58 \mathrm{~b}$ & $24.58 \mathrm{c}$ \\
\hline $75 \mathrm{~N}+\mathrm{M}$ & $14.20 \mathrm{a}$ & $14.12 \mathrm{a}$ & $13.84 \mathrm{a}$ & $13.86 \mathrm{a}$ & $0.76 \mathrm{a}$ & $0.67 \mathrm{a}$ & $0.53 \mathrm{a}$ & $0.54 \mathrm{a}$ & $334.2 \mathrm{a}$ & $359.0 \mathrm{a}$ & $28.33 \mathrm{a}$ & $28.42 \mathrm{a}$ \\
\hline LSD 0.05 & 0.42 & 0.28 & 0.42 & 0.28 & 0.024 & 0.016 & 0.266 & 0.017 & 12.5 & 4.8 & 0.19 & 0.14 \\
\hline $\begin{array}{c}\text { Interaction } \\
\mathbf{V} * \mathbf{F}\end{array}$ & $\mathrm{ns}$ & $*$ & ns & $*$ & $* * *$ & $* * *$ & $* * *$ & $* * *$ & $* * *$ & $* * *$ & $* * *$ & $* * *$ \\
\hline
\end{tabular}


J. Adv. Agric. Res. (Fac. Agric. Saba Basha)

Table(10): Effect of nitrogen, bio-fertilization and wheat cultivars on milling character.

\begin{tabular}{|c|c|c|c|c|c|c|}
\hline & \multicolumn{2}{|c|}{ flour } & \multicolumn{2}{|c|}{ bran } & \multicolumn{2}{|c|}{ Short } \\
\hline & Session1 & Session2 & Session1 & Session2 & Session1 & Session2 \\
\hline \multicolumn{7}{|c|}{ Varieties $(\mathbf{V})$} \\
\hline V1 & $72.03 \mathrm{a}$ & $72.17 \mathrm{a}$ & $21.72 \mathrm{a}$ & $22.01 \mathrm{a}$ & $6.7 \mathrm{~b}$ & $6.6 \mathrm{~b}$ \\
\hline $\mathbf{V 2}$ & $71.72 \mathrm{ab}$ & $72.03 \mathrm{a}$ & $20.17 \mathrm{c}$ & $20.26 \mathrm{c}$ & $7.5 \mathrm{a}$ & $7.3 \mathrm{a}$ \\
\hline V3 & $71.57 \mathrm{~b}$ & $71.93 \mathrm{a}$ & $20.82 \mathrm{~b}$ & $20.81 \mathrm{~b}$ & $7.6 \mathrm{a}$ & $6.7 \mathrm{~b}$ \\
\hline LSD 0.05 & 0.45 & 0.45 & 0.26 & 0.07 & 0.22 & 0.44 \\
\hline \multicolumn{7}{|c|}{ Fertilization $(\mathbf{F})$} \\
\hline $\mathbf{0} \mathbf{N}$ & $75.3 \mathrm{a}$ & $75.5 \mathrm{a}$ & $17.38 \mathrm{f}$ & $17.46 \mathrm{f}$ & $7.31 \mathrm{~b}$ & $7.0 \mathrm{ab}$ \\
\hline $35 \mathrm{~N}$ & $72.7 \mathrm{c}$ & $73.27 \mathrm{c}$ & $19.87 \mathrm{~d}$ & $19.91 \mathrm{~d}$ & $7.34 \mathrm{~b}$ & $6.81 \mathrm{bc}$ \\
\hline $75 \mathrm{~N}$ & $69.48 \mathrm{e}$ & $69.80 \mathrm{e}$ & $23.12 \mathrm{~b}$ & $23.24 \mathrm{~b}$ & $7.38 \mathrm{ab}$ & $6.9 \mathrm{ab}$ \\
\hline 0N+Microbein & $74.4 \mathrm{~b}$ & $74.5 \mathrm{~b}$ & $18.03 \mathrm{e}$ & $18.37 \mathrm{e}$ & $7.5 \mathrm{a}$ & $7.12 \mathrm{a}$ \\
\hline $35 \mathrm{~N}+\mathrm{M}$ & $70.17 \mathrm{~d}$ & $70.27 \mathrm{~d}$ & $22.65 \mathrm{c}$ & $22.78 \mathrm{c}$ & $7.16 \mathrm{c}$ & $6.93 \mathrm{ab}$ \\
\hline $75 \mathrm{~N}+\mathrm{M}$ & $68.47 \mathrm{f}$ & $68.8 \mathrm{f}$ & $24.37 \mathrm{a}$ & $24.38 \mathrm{a}$ & $7.14 \mathrm{c}$ & $6.7 \mathrm{c}$ \\
\hline LSD 0.05 & 6.34 & 0.22 & 0.33 & 0.13 & 0.12 & 0.17 \\
\hline $\begin{array}{c}\text { Interaction } \\
\mathbf{V} * \mathbf{F}\end{array}$ & $* * *$ & $* * *$ & $* * *$ & $* * *$ & $* * *$ & $* * *$ \\
\hline
\end{tabular}

\section{REFERENCES}

AACC.(2000). American Association of Cereal Chemists. Cereal Laboratory Methods. St. Paul., Minnesota, USA.

AOAC. (2000). Official methods of analysis of the Association of Official Analytical Chemists.

Abd- El- Rahim, E.A. (1999). Balady bread prepared from wheat flour mixed with $20 \%$ corn flour, the problem and solution. Proceeding of international conference and exhibition for food industries quality control compassable, December 1-3, Alexandria, Egypt.

Abd-El-Razik, M.A. El- shaarawy, (2013). Evolution of some new cultivars under agricultural treatments. Ph.D. Thesis Fac. Agric. Al-Azharuniy., Egypt.

Ashmawy,F., M.S. El-Habal, H.S. Saudy and Iman, KAbbas (2010). The relative contribution of yield of some wheat cultivars grown under different nitrogen fertilizer levels. Argon. Dept., Fac. Agric., Ain Shams Univ. Egypt. J. Agric. Res., 88 (1). 99-105

Chapman, H.D. and P.F. Pratt, (1978). Method of Analysis for Soil and Water. $2^{\text {nd }}$ Ed., Chapter, 17pp 150-161.Uni. Calif. Div. Agric. Sci. USA.

EI - Naggar, H.M.M. (2003). Varietal response and of yield components Chemical composition of wheat grains to nitrogen sources. Annals of Agric. Sic. Mashtohor, 35(2):731-744.

FAO. (2015). WWW. Fao.org /ag/ AGP/ AGPC/ doc/ field/ wheat/ Africa/Egypt legyptagec.htm. 
Gafarr, N. A. (2007). Response of some bread wheat varieties grown under different levels of planting density and nitrogen fertilizer. Minufiya J. Agric. Res 32:165-183

Gomez, A. K. and A.A. Gomez, (1984). Statistical procedures for agricultural research. ( $2^{\text {nd }}$ edition). John Wiley and Sons. New York.

Kandil, A.A., M.H. El-Hindi, M.A. Badawi, S.A. El-Morarsy and F.A.H.M. Kalboush, (2011). Response of wheat rates of nitrogen, bio-fertilizers and land leveling. Crop Environ., 2:46-51.

Mahrous, M. A. and Y. A. Abd-Elhady (2006). Studies of quality and baking traits in bread wheat. Minufiya. J. Agric. Res., 31:899-914.

Rahimi, A., (2010). Effect of potassium and nitrogen on yield components of dry land wheat in Boyerahmad Region of Iran .Ann. Biol. Res., 3:3274-3277.

Seleiman, M.F., S.M. Abdel - Aal, M.E. Ibrahim and P. Monneveux (2010). Variation of yield, milling,technological and rheological characteristics in some Egyptian bread wheat (Triticum aestivum.,L.) Cultiva rs .Egypt. J. Food. Agric. Sic, Minufiy Univ., 22(2):84-90.

Toaima, Amal, A.El-Hofi and H. Ashoush. (2000). Yield and technological characteristic of some wheat varieties as affected by $\mathrm{N}$-fertilizer and seed rate .J. Agric. Sci. Mansoura Univ., 25(5):2449-2467.

Waller, R.A. and D.B. Duncan, (1969). A bays rule for the symmetric multiple comparison problem. Amer. State. Assoc. j. Dec.: 1485-1503 (C.F.CD Rom Computer System).

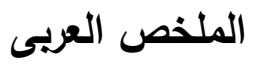

تأثير التسميد النيتروجينى والحيوى على المحصول والصفات التكنولوجيه لبعض

اصناف القمح

$$
\begin{aligned}
& \text { (')فتحى ابراهيم رضوان ، (')محود عبدالعزيز جمعه ، ('امحد عبدالحميد زيتون ، (1) }
\end{aligned}
$$

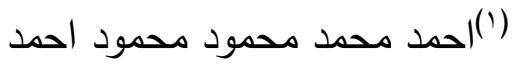

$$
\begin{aligned}
& \text { (') قسم الإنتاج النباتي - كلية الزراعة (سابا باشا) - جامعة الاسكندرية }
\end{aligned}
$$

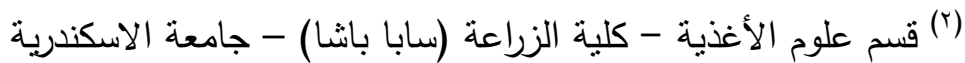

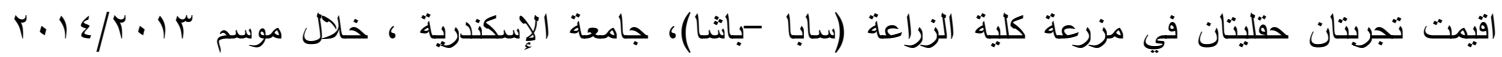

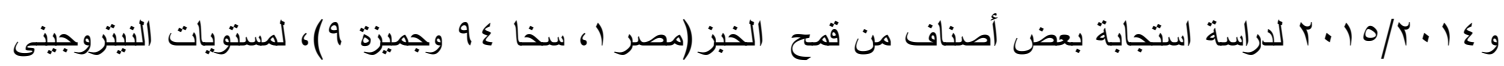

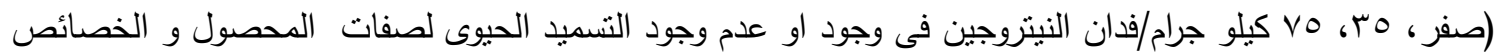

Vol. 20(3), 2015 
التكنولوجية من الدقيق باستخدام تصميم القطع المنشقة مره واحده فى ثلاثة مكررات. وكان المحصول السابق هو القطن

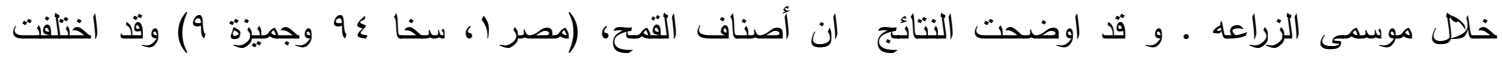

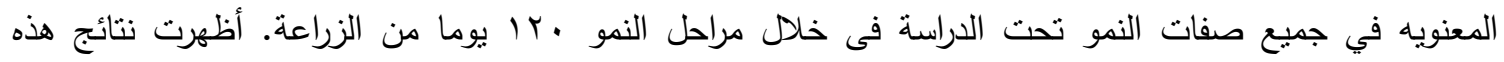

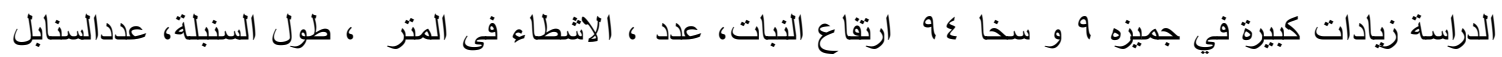

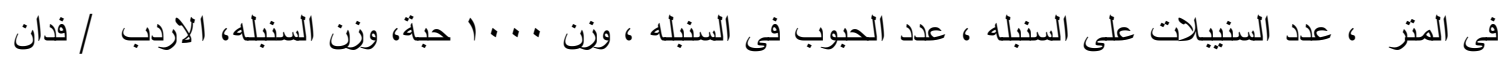

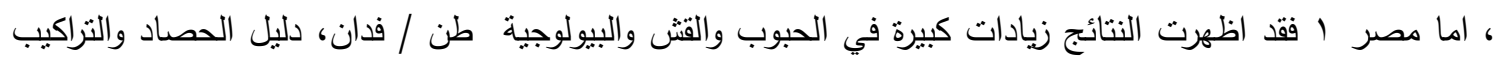
الكيميائية في رطوبة القمح٪، الرماد٪، رقم السقوط، الوزن النوعى ، ومجموع البروتين٪، الجلوتين الرطب٪، الجلونين الجاف\%، دليل الجلوتين٪ وصفات الدقيق التكنولوجية في الرطوبة الدقيق٪، الرطوبة النخاله٪، رماد الدقيق٪؛ رمادالدقيق رقم السقوط ثانية، الدقيق الرطب الجلوتين٪ وصفات الطحن ٪ خلال الموسمين. مستويات النيتروجين والأسمدة الحيوية أنتجت زيادات كبيرة في جميع صفات النمو والتركيب الكيميائي. أظهر التقاعل بين أصناف القمحتح، ومستويات النيتروجين والأسدة الحيوية والهامة لجميع الصفات المدروسة. وأظهرت النتائج التي تم الحصول عليها على أعلى ارتفاع النبات، وعدد من الاشطاء ، وطول السنبلة، عدد السنابل المتز المربع ، وعدد من بالسنبيلة /السنبله، عدد السنابل النواة، وزن ..اعبة، وزن السنبله، الاردب ، محصول الحبوب، القش والبيولوجية العائد طن / فدان،

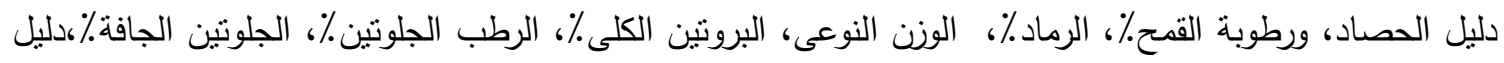

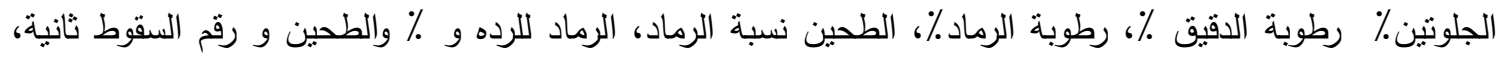

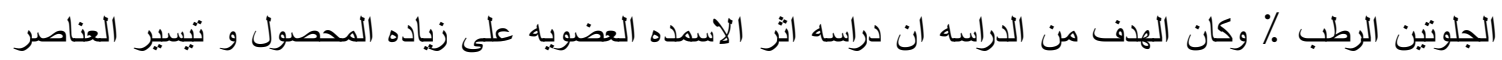

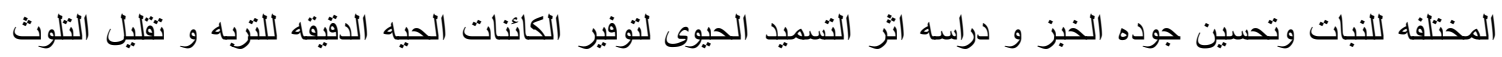
وزياده الخصائص التكنولوجيه للقمح و دراسه افضل صنف ذو خواص تكنولوجيه جيده و دراسه التفاعل بين الاصناف و التسميد العضوى والحيوى على انتاجيه القمح وجودته. 
J. Adv. Agric. Res. (Fac. Agric. Saba Basha) 